\title{
A Novel Portfolio Optimization Model Based on Pattern Recognition Algorithms
}

\author{
Gongbo Tan $^{1, \text { a }}$, Yan Wang ${ }^{2, b}$ and Jun Xiong ${ }^{3, c}$ \\ ${ }^{1}$ School of Economics and Management, Beijing University of Posts and Telecommunications, \\ Beijing,1000876, China \\ ${ }^{2}$ College of Mathematics and Computer Science, Hunan Normal University, Changsha, 410000, \\ China \\ ${ }^{3}$ School of Management, Lancaster University, Lancaster,LA1 4YW, UK \\ ajamestan9574@gmail.com, b wangyan1995000@sina.com, cj.xiong@lancaster.ac.uk
}

Keywords: multi-criteria unbiased optimization, Particle swarm optimization, Artificial Intelligence, genetic algorithm .

\begin{abstract}
There are two types of modern portfolio theory: narrow and broad. A narrowly conceived portfolio theory is often referred to by the name Markowitz portfolio theory. Often generalized portfolio theory, in addition to classical portfolio theory, and various alternative portfolio theories, include the capital asset pricing model and an effective securities market theory of capital markets. At the same time, because the traditional EMH cannot explain market anomalies, the various portfolio theories are have been challenged by behavioral financial theory. This study uses pattern recognition algorithms to improve the traditional portfolio optimization models. By utilizing improved particle swarm optimization algorithms, a hybrid optimization model based on this would be the result of the experiment, compared with that based on the traditional neural network model for quantitative model effect. Particle swarm optimization algorithms were originally generated to produce graphical simulations of flocks of birds and other such unpredictable movements. The developmental basis of the algorithms used in this experiment are observations of animal social behavior, demonstrating that sharing of information society provides groups with an evolutionary advantage. By joining near the speed of matching, taking multidimensional search into account, and according to speeds and distances, we can thus form the original version of particle swarm optimization. After introducing the parameters of inertia weight to better control the development of swarm behavior, we have produced the standard version. This study will focus on analysis to improve the process of generating particle swarm optimization models.
\end{abstract}

\section{Introduction}

A stockbroker weighing a trade will be considering not only the merits of the investment itself, but also its merits in relation to all other potential trades available at that moment. The complexity of this problem calls for an algorithm within the framework of multi-criteria unbiased optimization (MUO,) which can produce an "ideal" trading strategy in the face of a complex range of possible targets. Timing and speed are critical components of trading success. Historically, stockbrokers have relied on two techniques: primary examination, which examines the fundamentals of an asset to in an attempt to determine its future performance, and specialized investigation, which ignores the underlying properties of an asset and instead dissects its trading history through outlines and scientific equations called specialized pointers [1]. More recently, Artificial Intelligence (AI) techniques have also been used, generally in the second category of analysis. There are two primary roles for AI in securities trading [2]. To begin with, it can be used as an instrument to build up an exchanging framework whose objective is to give trading signals using authentic end of day business sector information. This type of algorithm can quickly synthesize information and present it to a human operator, but will not itself execute any trades. Artificial Intelligence can also be used actively, however, as a component of automated trading software whose goal is to post buy and 
offer requests which are prepared by a dummy trade. These algorithms more often than not use information progressively, and are approved by the specialist's interest in a mimicked stock exchanging trade. They primarily use models such as hereditary calculation and neural systems to weigh returns in equities markets. There are also algorithms that are designed to optimize given parameters based on specialized markers. For our research, the parameters were set according to the markets generated by a metaheuristic computation. We used these heuristics to advance the parameters of three specialized markers [3].

Those using AI algorithms in the second category can refer to studies in which software operators rely on composite exchanging rules prepared by metaheuristic calculations and genetic programming. The operators were generally matched against both purely AI traders and traders unassisted by any sort of software. The results of this series of studies showed AI assisted trading to be strikingly successful, but it should be noted that this success was correlated with a narrowly defined set of parameters, as opposed to the true complexity of the securities market. Be that as it may, it is entirely possible that these types of algorithms could be used commercially, given that a trading strategy based around a narrow success criteria can still be profitable. Additionally, dealers give diverse significance to these components, contingent upon their exchanging identities, for example the amount of risk they take. This requires an instrument that can accurately weigh different types of risk and reward [4]. Of course, this is precisely what a multi-criteria unbiased optimization framework is designed to do. A few uses of multi-criteria unbiased optimization already exist at the time of this writing in the fields of stock prediction and analysis, portfolio determination, and portfolio optimization. In addition, researchers have made use of multi-criteria unbiased optimization through high frequency trading in money markets. Researchers utilized a metaheuristic calculation based on multi-criteria unbiased optimization as a way to deal with high frequency trading software based on two parameters, to achieve specific benefit proportion and change of benefit. The software was tested by executing trades on a dummy business sector test platform [5]. Utilizing this approach, the software eventually developed a few trading strategies which were superior, at least in terms of the narrowly defined success parameters, to the strategies deployed by unassisted human traders. Many unbiased observers were delighted with this result, as there has been persistent demand for accurate, synthesized end of day business information throughout the industry. This is an element of particular emphasis for the authors of this paper.

Recently, another methodology for optimization of Conditional Value at Risk (expected shortfall) was recommended and used in a few applications. For consistent dispersions, Conditional Value-at-Risk is characterized as the normal shortfall surpassing Value at Risk (VaR). For the most part, Conditional Value-at-Risk is the weighted normal of Value at Risk and shortfall surpassing Value at Risk. Critical to the methodology is an optimization system for computing Value at Risk and simultaneously enhancing Conditional Value at Risk. The purpose of extending the composition in this way to deal with the optimization issues with Conditional Value at Risk imperatives. Specifically, the methodology can be utilized for expanding expected returns under conditional Value at Risk imperatives [6][8]. Numerous Conditional Value at Risk imperatives with different certainty levels can be utilized to shape the profit/shortfall dispersion. The study develops the Conditional Value at Risk minimization approach and the capacities of Conditional Value at Risk at different variable levels. Analysis demonstrates that this methodology can also be utilized to maximize profit capacities for the occurrence of returns under Conditional Value at Risk limitations, instead of minimizing Conditional Value at Risk in general. It is also possible to force many Conditional Value at Risk requirements with various certainty levels and shape the shortfall circulation as per the inputs specified. These inclinations are indicated specifically in percentile terms, in contrast with standard methodology, which determines hazard inclinations in terms of utility capacities [7].

The researchers detail the portfolio determination as a tri-target optimization issue in order to discover trade-offs between risk, return and the quantity of securities in the portfolio. Amount and class limitations are also brought into the model, keeping in mind the end goal of constraining the extent of the portfolio, maintaining assets with regular qualities, and keeping a strategic distance 
from minor assets [9]. Since the proposed portfolio determination model includes blended whole number choice variables along with multivariable targets, it is a complicated matter to find the optimal composition. In any case, for investing purposes it will typically be satisfactory to find an effective platform which furnishes the financial specialist with a differing set of portfolios containing all conceivable exchange offs between the destinations within restricted computational time.

The study was displayed within a remote currency speculation structure including the expectation of outside monetary trading rates and the portfolio optimization under certain constraints. This research tests two machine learning strategies, vector algorithms and neural systems, and also the customary trading strategy, to predict the trading movements for three foreign currencies including the Dollar, the Euro, and the Franc. Taking into account these forecasts, researchers pick two out of the three monetary forms recorded above and assemble a portfolio by embracing multi-target portfolio optimization methods, which expand the probability of gains while minimizing risk [10-12].

In particular, analysis shows a stock exchanging framework that uses multi-criteria unbiased molecule swarm optimization (MUPSO) with specialized monetary markers. Utilizing end-of-day business-sector information, the framework improves the weights of a few specialized markers by more than two percent target capacities. [13].

The Sharpe proportion of the final portfolio bears out this optimistic analysis. The proposed multi-criteria unbiased optimization algorithm depends on Particle Swarm Optimization as opposed to on hereditary calculation for two reasons. To begin with, the optimization variables that are weighted are constant, and for this reason Particle Swarm Optimization fitting strategy doesn't necessitate discretization of the choice variables in the same way that metaheuristic calculation does. Also, the researchers assert that Particle Swarm Optimization and hereditary calculation, on the whole, yielded the same type of portfolio arrangements; in any case, Particle Swarm Optimization has the advantage of being more computationally productive than metaheuristic calculation. Hence, utilizing Particle Swarm Optimization could be an incredibly advantageous technique, since it could accelerate calculation time, a critical element in developing a stock trading algorithmic framework [14]. The researchers did in fact claim that that enhancing the calculation time was a potential test variable in the course of the study. Researchers in this way have shown one of only a handful of genuine uses of multi-criteria unbiased molecule swarm optimization in practice. This is critical particularly if analysts consider that the growing number of types of the multi-variable unbiased molecule swarm optimization calculation corresponds to a growing acceptance of multi-criteria unbiased molecule swarm optimization's viability in solving practical problems [15].

\section{Related Work- Multi-Variable Unbiased Optimization by means of Particle Swarm Optimization}

In this field, our research gives a snapshot of issues with multi-criteria unbiased optimization and molecule swarm optimization calculations. A brief summary of tests of Particle Swarm Optimization is necessary to comprehend multi-criteria unbiased optimizations and the utilization of multi-variable unbiased molecule swarm optimization in practical applications. Thus, researchers present Multi-target Particle Swarm Optimization Crowding Distance, the Particle Swarm Optimization based multi-criteria unbiased optimization technique that analysts will use in the development of the trading system.

Multi-criteria optimization issues are not simply restricted to single targets. Many times, they require having parity between various interacting and sometimes contradictory elements. The optimization techniques in question addresses this issue. Multi-criteria unbiased optimization involves finding an arrangement of elements that enhances a limited number of objectives. An ideal algorithm could therefore give a diverse range of results, at least in contrast to the results given by a single target arrangement, since what is required is an arrangement of trading strategies as opposed to a solitary universal ideal. This idea is expressed in the concept of Pareto optimality (PO). The variable result can thus be expressed as follows: 
To simplify,

The focus is thus on

$$
\begin{gathered}
\mathrm{b}=\mathrm{s}(\mathrm{a}) \\
\mathrm{s}(\mathrm{a})=\{\mathrm{s} 1(\mathrm{a}), \ldots, \mathrm{sx}(\mathrm{a})\}
\end{gathered}
$$

where,

$$
\begin{aligned}
\mathrm{t}(\mathrm{a})=\{\mathrm{t} 1(\mathrm{a}), \ldots, \mathrm{tx}(\mathrm{a})\}<0 \\
\mathrm{u}(\mathrm{a})=\{\mathrm{u} 1(\mathrm{a}), \ldots, \mathrm{ux}(\mathrm{a})\}=0
\end{aligned}
$$

$\mathrm{a}=(\mathrm{a} 1, \ldots$, ay) is an element of $\mathrm{A}$

$\mathrm{b}=(\mathrm{b} 1, \ldots, \mathrm{bx})$ is an element of $\mathrm{B}$

Here, "a" is known as a choice vector, "A" is the choice, y is a goal vector and "B" is the integrated goal, while $\mathrm{t}(\mathrm{a})$ and $\mathrm{u}(\mathrm{a})$ are requirements that must be fulfilled during the optimization procedure. The result of the above procedure is the Pareto frontier which comprises one or more Pareto efficient outcomes, which are choice vectors "a" that advance a set of distinctive destinations. Pareto optimality depends on the idea of predominance. Our research shows that competitor arrangement $\mathrm{a}$ (c) commands another hopeful arrangement $\mathrm{a}(\mathrm{d})$ when both of the accompanying conditions are fulfilled. To begin with, $a(d)$ is no less advantageous than $x(d)$ in every goal capacity; and second, a(d) is entirely superior to anything $\mathrm{x}(\mathrm{d})$ in no less than one target capacity. In many unbiased tests, the ideal arrangement is the arrangement of all non-ruled arrangements. A nonoverwhelmed arrangement is known as a Pareto point although an arrangement of all Pareto efficient points is known as a Pareto frontier.

Molecule Swarm Optimization is tested in this model. Molecule Swarm Optimization is a wellknown computational method that is modeled on the behavior of nesting swallows, or other flocking animals. Researchers have two explanations for Particle Swarm Optimization's success. Firstly, it relies on relatively simple operational inputs, so its execution is direct. Secondly, it has been shown to have an exceptionally broad range of applications, delivering generally accurate results at using relatively little processing power. In particular, Molecule Swarm Optimization has demonstrated success in solving optimization problems with large numbers of possible outcomes. Our analysis shows that while Particle Swarm Optimization's execution is practically identical to metaheuristic calculation, Particle Swarm Optimization is computationally more efficient than metaheuristic calculation. In Particle Swarm Optimization, a collection of conceivable arrangements called elements are initially defined. The elements are programmed to solve a relatively simple task, or hold a relatively simple position in relation to the other elements. Every element, or molecule, maintains an optimal arrangement it has discovered and by extension the arrangement that the so called called molecule swarm has "discovered" will generate a complex outcome that will be optimal for the group as a whole. The course of the pursuit is then redesigned in light of the estimations of molecular elements and the gathering as a whole will achieve efficiency. The position and speed improvements achieved by Particle Swarm Optimization are demonstrated as follows: By treating the molecule as an individual element with impact only on its direct neighbors, its velocity can be calculated by taking the result of the particles latency alongside the speed at the current time. This is added to the momentum of the element, which is then duplicated and compounded by the degree of certain irregular numbers drawn from uniform circulation. The molecule's position is given by extrapolation of the position condition at a given time to the speed that is obtained from the cycle time $\mathrm{t}+1$.

\section{Multi-Criteria Unbiased Optimization as Applied in Financial Markets}

The following segment depicts our trading framework, and demonstrates how it utilizes the features of multi-variable unbiased optimization.

The ultimate goal of our project has been to develop a trading platform. Our research thus upgraded an arrangement of weights connected with defined targets. We utilized metaheuristic calculation to upgrade a weighted blend of four markers. In one analysis, we utilized the Sharpe 
Ratio as our goal capacity. The trading platform also enhanced an arrangement of weights connected with the defined targets; in addition they were improved by more than two target capacities utilizing a multi-criteria unbiased optimization strategy. Specialists have often chosen percent return and Sharpe proportion as the ultimate target indicators. The Sharpe Ratio is characterized as the proportion of normal returns weighted against risk. The percent return and the Sharpe Ratio measure two different trading criteria: benefit and risk. A trading framework with a high return may, for instance, bring an undesirable degree of risk into the trade. The Sharp Ratio is generally defined as follows:

Sharpe Ratio $=$ the mean estimation of all profits, that is the return or loss of a trading framework divided by the standard deviation of all returns $\}$

This passage will discuss trading strategies. Every specialized marker was connected with a standard trading guideline characterized in writing. The trading principle connected with a target produced a sign quality, unity was provided if the pointer was in a long location and an estimation of unity that is negative was provided if the marker was in a Short location. In addition, a weight was fixed to every specialized pointer. The trading decision relied on the weighted decision's value, which is the simply the sum of sign quality and weight. A trade was executed in the event that this quality surpassed 0.5. A trade was cancelled when this value dropped below 0.5; that is the sum of sign quality alongside weight. The weighted choice system additionally judged the sum worthy of being utilized as a part of the trade. An underlying venture quality was utilized to exchange the standard. True benefits were not realized, since the performed trades were not utilized for reinvestment. Trading costs were excluded in the outline of this framework.

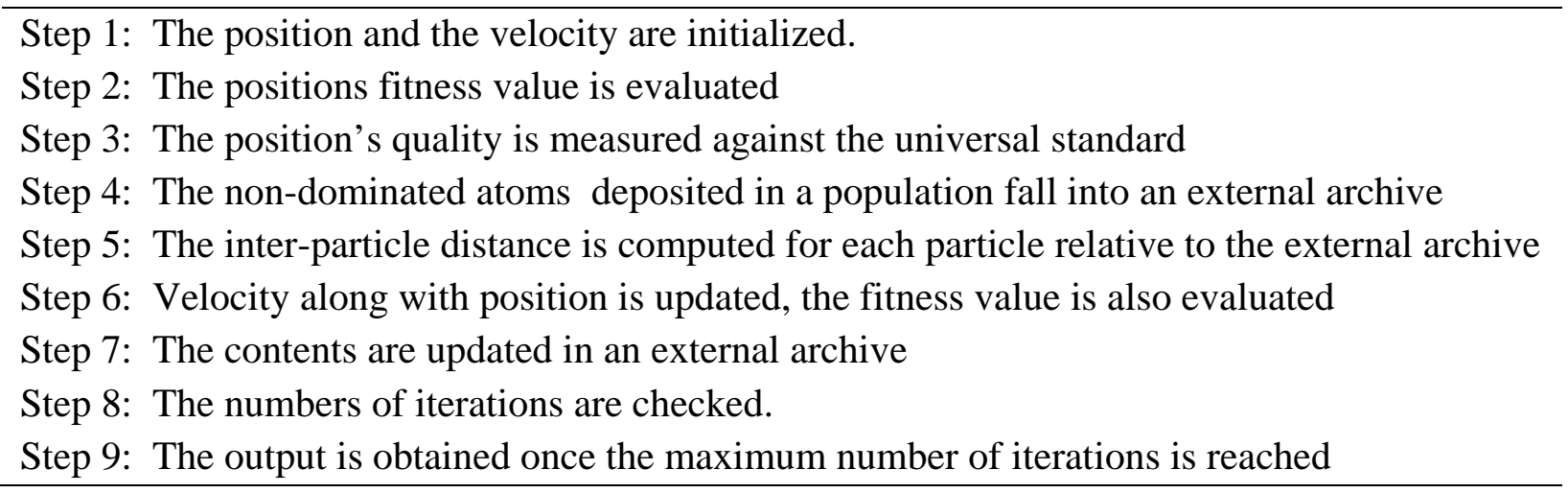

Figure 1. The multi-criteria unbiased particle swarm optimization -CD algorithm

\section{Training and testing}

Our analysis used the non-optimal area under the Pareto frontier as the default orientation opinion, and so contrasted our general execution in contradiction of the alternate markers. This area is generally known as the bivariate middle of the extent. It is demonstrated in the diagram with a red reference bullet.

Population magnitude and quantity of eras analyzed differed based on the estimations of the populace magnitude and quantity of eras, both ranges steadily between 100 and 600, while keeping alternate restrictions steady. The testing then continued for a period. We exhibit our results in Figure 2 and figure 3. From the figures we are able to see that the populace measure that returned advanced percent returns is 150 . Depending on the trading variables specified, we were able to pick either 100 or 600 for the populace size. If the operator chose to seek higher returns, they would set the populace size to 100 . After this decision, picking the quantity of eras to utilize is somewhat simpler, as the researcher will have set the populace size of 100. 


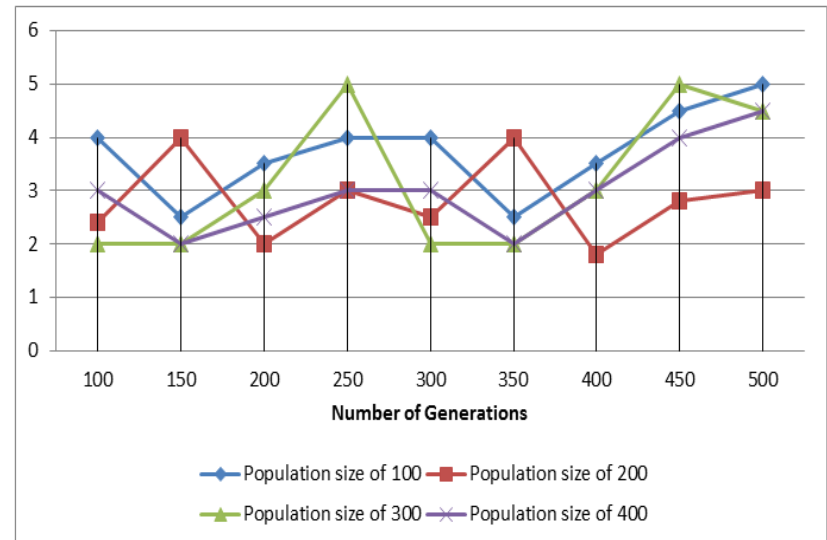

Figure 2. Graph showing the average value of the return

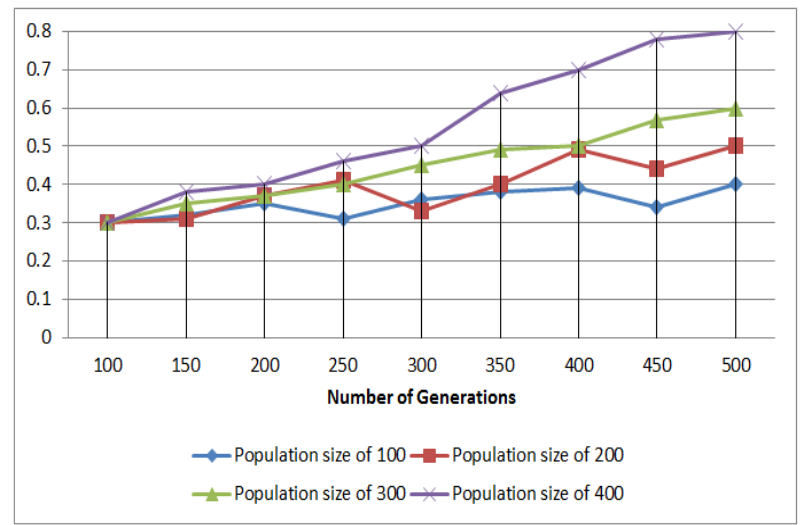

Figure 3. The average Sharpe Ratio across trades

\section{Conclusion}

This study has introduced a securities trading framework that uses an algorithm for multi-target molecule swarm optimization. Utilizing authentic end-of-day business sector information, the framework used the trading signals from an arrangement of monetary specialized markers with the specific end goal of building up a trading standard which is optimized for two target capacities: the Sharpe Ratio and the percent return. The framework performed well using both prepared and outof-test information. In terms of return percentages, the framework outperformed the control group on all occasions, in a few rounds it even out-played out the business sector itself. In terms of the Sharpe Ratio, the framework reliably achieved fundamentally superior results compared to all of the control groups. These outcomes demonstrate the capability of the theorized framework as a piece of software designed to settle securities trades, and can be adapted for further optimization. Amongst the upgrades that might be developed in the future are the development of more sophisticated trading criteria, the application of our model to different types of trades, for example, longer term investing, and then the eventual use of our model in radically different types of markets.

\section{References}

[1]A. Abraham, C. Grosan, S. Y. Han, A. Gelbukh, Evolutionary multiobjective optimization approach for evolving ensemble of intelligent paradigms for stock market modeling, in: Lecture Notes in Computer Science, 4th Mexican International Conference on Artificial Intelligence (MICAI'05), vol. 3789, Springer Verlag, Germany, 2005, pp. 673-681.

[2]R. Armananzas, J. A. Lozano, A multiobjective approach to the portfolio optimization problem, in: Proceedings of the 2005 IEEE Congress on Evolutionary Computation (CEC 2005), vol. 2, 2005, 


\section{IEEE Press.}

[3]D. de la Fuente, A. Garrido, J. Laviada, A. Gfiomez, Genetic algorithms to optimise the time to make stock market investment, in: GECCO '06: Proceedings of the 8th annual conference on Genetic and evolutionary computation, ACM Press, New York, NY, USA, 2006.

[4]A. Fernandez, S. Gomez, Portfolio selection using neural networks, Computers and Operations Research 34 (2007) 1177.

[5]S. Janson, D.Merkle, M. Middendorf, Molecular docking with multi-objective particle swarm optimization, Appl. Soft Comput. 8 (1) (2008) 666-675.

[6]J. Li, S. Taiwo, Enhancing _nancial decision making using multi-objective financial genetic programming, in: Proc.of 2006 IEEE Congress on Evolutionary Computation (CEC'06), Sheraton Vancouver Wall Centre, Vancouver, BC, Canada, 2006.

[7]Y. Niu, L. Shen, The optimal multi-objective optimization using pso in blind color image fusion, mue 00 (2007) 970-975.

[8]M. R. Sierra, C. C. Coello, Multi-objective particle swarm optimizers: a survey of the state-ofthe-art, Int. J. Comput. Intell. Res. 23 (2006) 287-308.

[9]Liu, Y. J., \& Zhang, W. G. (2015). A multi-period fuzzy portfolio optimization model with minimum transaction lots. European Journal of Operational Research, 242(3), 933-941.

[10]Liu, Yong-Jun, and Wei-Guo Zhang. "Fuzzy portfolio optimization model under real constraints." Insurance: Mathematics and Economics 53, no. 3 (2013): 704-711.

[11]Mansini, R., Ogryczak, W., \& Speranza, M. G. (2015). Portfolio Optimization with Transaction Costs. In Linear and Mixed Integer Programming for Portfolio Optimization (pp. 47-62). Springer International Publishing.

[12]Hu, Wenbo. "Calibration of multivariate generalized hyperbolic distributions using the EM algorithm, with applications in risk management, portfolio optimization and portfolio credit risk." (2016).

[13]Mansini, Renata, Włodzimierz Ogryczak, and M. Grazia Speranza. "Portfolio Optimization with Other Real Features." In Linear and Mixed Integer Programming for Portfolio Optimization, pp. 63-72. Springer International Publishing, 2015.

[14]Yang, Ze-Bin, Rong-Xi Zhou, Qiang Zhang, and Mei Yu. "A Portfolio Optimization Model Based on Information Entropy and Fuzzy Time Series." In Business Intelligence and Financial Engineering (BIFE), 2013 Sixth International Conference on, pp. 166-170. IEEE, 2013.

[15]Konno, H., \& Yamazaki, H. (1991). Mean-absolute deviation portfolio optimization model and its applications to Tokyo stock market. Management science, 37(5), 519-531. 